\title{
Hommage au Professeur Claude Chastel (1928-2018)
}

\section{Tribute to Professor Claude Chastel (1928-2018)}

\section{Y. Buisson · V. Deubel}

Reçu le 12 mars 2019; accepté le 14 mars 2019

(C) Société de pathologie exotique et Lavoisier SAS 2019

Le Professeur Claude Chastel, est décédé le 24 décembre 2018 à l'âge de 90 ans (Figs 1 et 2).

Né à Toulon en 1928, il est docteur en médecine (Ecole du service de santé militaire de Lyon, 1952) et diplômé de l'Institut Pasteur en microbiologie et immunologie (grand cours).

Claude Chastel a une longue expérience du milieu tropical puisqu'une partie de sa carrière s'est déroulée au Viêtnam, au Cambodge et en Guinée. En 1961, il crée à l'Institut pasteur de Phnom Penh, le Service de Virologie Humaine et d'Entomologie Médicale, premier laboratoire d'arbovirologie situé hors de France. Ce sont 5 années très fructueuses au cours desquelles il isole le virus Chikungunya (1961), divers sérotypes du virus de la dengue (1962), les virus Getha et de l'encéphalite japonaise (1966). Il découvre un nouvel arbovirus chez la chauve-souris, baptisé «Phnom Penh bat » et travaille également sur les virus de la poliomyélite et de la rage humaine.

On le voit (Fig. 3) entouré du personnel de son laboratoire. Tous ont disparu en 1975, massacrés par les Khmers rouges qui ont complètement détruit ce premier Institut Pasteur qui était situé sur la presqu'île de Chrui Chang War, entre le Mékong et le Tonlé Sap.

Professeur agrégé du Service de Santé des Armées de 1968 à 1972, praticien hospitalier, il et professeur des Universités, il prend en 1973 la direction du département de Microbiologie et hygiène à faculté de médecine et CHU de Brest.

Microbiologiste polyvalent, homme de terrain et de laboratoire, spécialiste de virologie et des maladies émergentes,

\footnotetext{
Y. Buisson

V. Deubel $(\bowtie)$

34 bld Heurteloup, 37000 Tours, France

e-mail : bulletin@pathexo.fr

Y. Buisson

* bulletin@pathexo.fr
}

Société de pathologie exotique, Hôpital de la Pitié Salpêtrière, Pavillon Laveran, 47/83 bld de l'hôpital, 75013 Paris, France

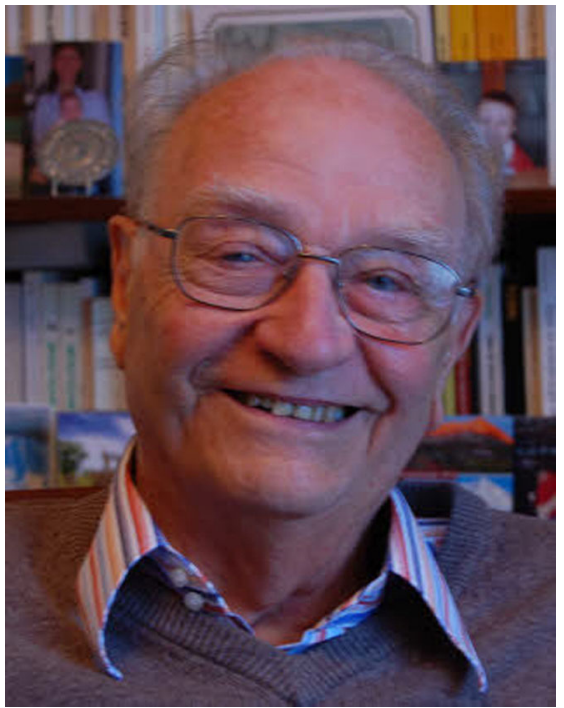

Fig. 1 Claude Chastel en 2000 / Claude Chastel in 2000

d'épidémiologie et d'histoire de la médecine, Claude Chastel laisse une œuvre scientifique considérable avec 277 publications recensées dans Medline, dont 51 parues dans le Bulletin de la Société de pathologie exotique (références cidessous).

Passionné par l'histoire des maladies infectieuses et écrivain de talent, Il a rédigé de nombreux ouvrages et présenté de nombreuses conférences sur le sujet; citons « Histoire des virus de la variole au sida » (1992), « Histoire des virus des origines à nos jours ", " Ces virus qui détruisent les hommes » (1996), « Virus émergents : vers de nouvelles pandémies?» (2006).

Il était consultant auprès de l'Organisation mondiale de la santé et membre correspondant de l'Académie de médecine.

Claude Chastel était officier de l'ordre de la Légion d'honneur et officier des Palmes académiques.

En outre, il était passionné de philatélie et fut le président du Club de philatélie brestoise de 1993 à 2008.

Avec lui, la SPE perd l'un de ses membres les plus attachants et les plus éminents. 


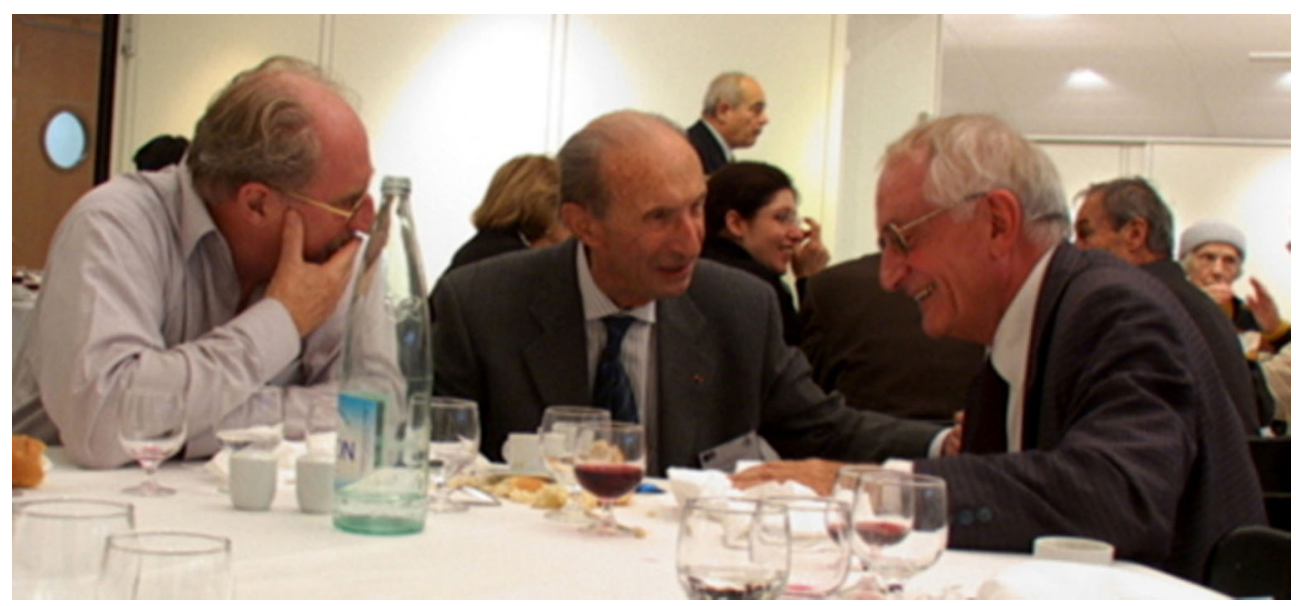

Fig. 2 Pierre Nozais, Guy Charmot et Claude Chastel à l'Institut Pasteur en 2005 / Pierre Nozais, Guy Charmot and Claude Chastel at the Institut Pasteur in 2005

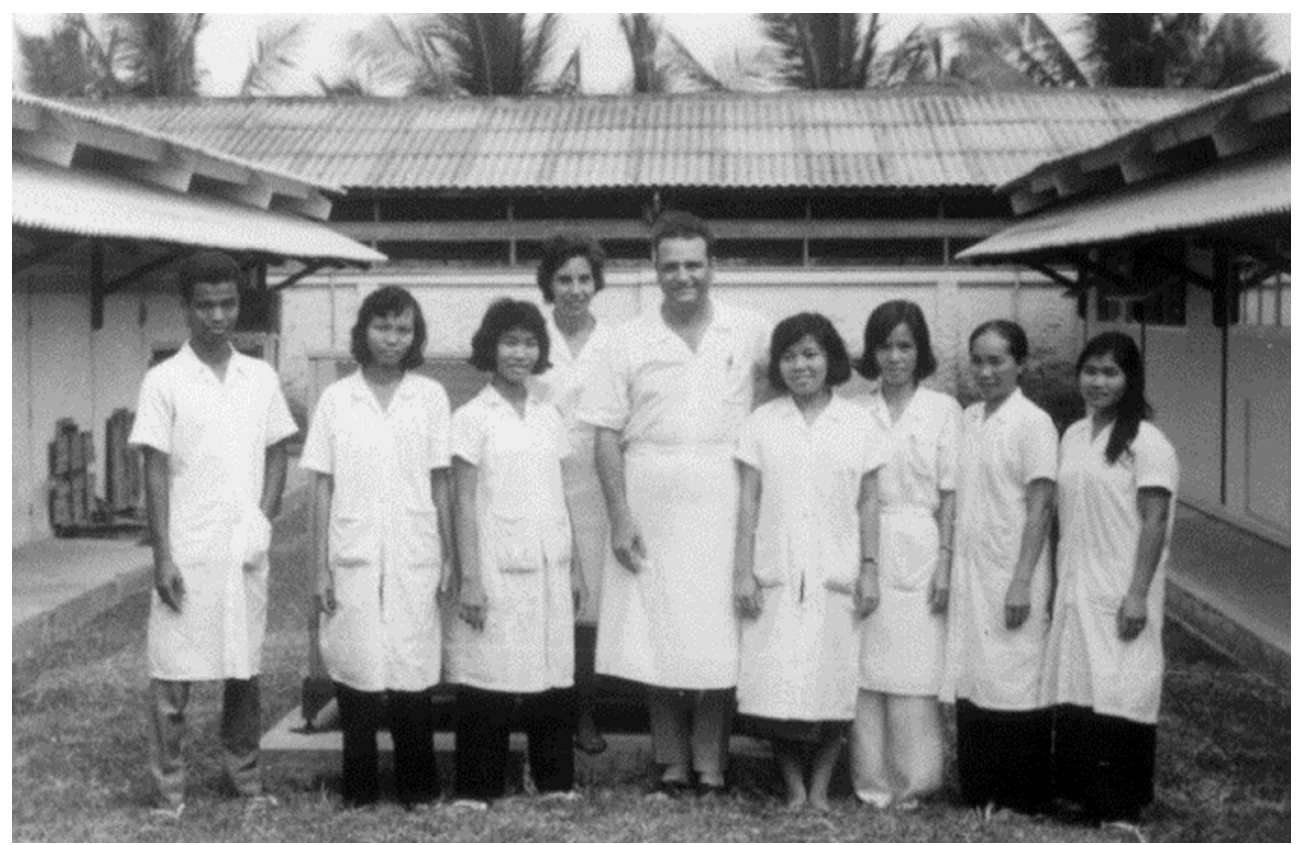

Fig. 3 Le Dr Chastel et son équipe à l'Institut Pasteur du Cambodge dans les années 1960 / Dr Chastel and his team at the Institut Pasteur of Cambodia in the $1960^{\text {th }}$

\section{Publications de Claude Chastel dans le Bulletin de la SPE (textes disponibles gratuitement en ligne sur http://www.pathexo. fr/standard-1863-1.html)}

Chastel C (2016) Anthropocène et viroses émergentes. Bull Soc Pathol Exot 109(3):207-9. doi: 10.1007/s13149-016-0503-4. Epub 2016 Jun 24 [http://www.pathexo.fr/documents/articles-bull/2016 109_207-209.pdf]

Chastel C (2014) Le "syndrome respiratoire du Moyen-Orient" (MERS) : qui est responsable, les chauves-souris ou le dromadaire ? Bull Soc Pathol Exot 107(2):69-73. doi: 10.1007/s13149-014-
0333-1. Epub 2014 Feb 15 [http://www.pathexo.fr/documents/articles-bull/131490069.pdf]

Chastel C, Chastel A (2013) À propos d'une note de 1926 sur un essai du BCG dans le traitement de la lèpre. Bull Soc Pathol Exot 106(2):126-30. doi: 10.1007/s13149-013-0280-2. Epub 2013 Feb 28 [http://www.pathexo.fr/documents/articles-bull/131490126.pdf]

Chastel C (2012) Quand certains Flavivirus remettent en cause nos certitudes. Bull Soc Pathol Exot. 105(4):251-5. doi: 10.1007/ s13149-012-0255-8. Epub 2012 Aug 24 [http://www.pathexo.fr/ documents/articles-bull/131490251.pdf]

Chastel C (2011) Infections inapparentes chez l'Homme : un cheval de Troie pour l'introduction et la diffusion des arbovirus transmis par des moustiques dans les régions non endémiques ? Bull Soc Pathol 
Exot. 2011 Aug;104(3):213-9. doi: 10.1007/s13149-011-0165-1. Epub 2011 Jun 21 [http://www.pathexo.fr/documents/articles-bull/ sprexot000142.pdf]

Tran A, Chastel C (2008) Grossesse et arbovirus transmis par des moustiques : conséquences pathologiques pour la mère et l'enfant. Une revue générale. Bull Soc Pathol Exot 101(5):418-24. [http:// www.pathexo.fr/documents/articles-bull/T101-5-3246.pdf]

Chastel C (2007) Le tsunami du 26 décembre 2004 : quel bilan épidémiologique deux ans plus tard ? Bull Soc Pathol Exot 100(2):139-42 [http://www.pathexo.fr/documents/articles-bull/T100-2-3035-4p.pdf] Le bureau de la SPE, avec la collaboration du Pr Cl. Chastel (2005) Le tsunami du 26 décembre 2004. Et maintenant ? Bull Soc Pathol Exot 98(1):3-4 [http://www.pathexo.fr/documents/articles-bull/T98-1editorial-2p.pdf]

Chastel C, Charmot G (2004) Épidémies bactériennes et virales d'origine zoonotique. Rôle de la chasse et du dépeçage d'animaux sauvages. Bull Soc Pathol Exot 97(3):207-12 [http://www.pathexo.fr/ documents/articles-bull/T97-3-2638-6p.pdf]

Chastel C (2003) Le centenaire de la découverte du virus de la fièvre jaune et de sa transmission par un moustique (Cuba 1900-1901). Bull Soc Pathol Exot 96(3):250-6 [http://www.pathexo.fr/documents/ articles-bull/T96-3-DK0.pdf]

Chastel C (2002) L'éradication mondiale de la poliomyélite en 2005 : progrès, priorités, problèmes. Bull Soc Pathol Exot 95(2):63-5 [http:// www.pathexo.fr/documents/articles-bull/T95-2-2413.pdf]

George JC, Chastel C (2002) Maladies vectorielles à tiques et modifications de l'écosystème en Lorraine. Bull Soc Pathol Exot 95(2):95-9 [http://www.pathexo.fr/documents/articles-bull/T95-2-2341.pdf]

Chastel C (2002) Léon Lapeyssonnie (1915-2001): le chercheur et l'enseignant. Bull Soc Pathol Exot. 95(5):310-3 [http://www. pathexo.fr/documents/articles-bull/T95-5-Chastel.pdf]

Chastel C (1999) Gertrude Belle Elion (1918-1999): chimiste de génie, découvreur des antiviraux modernes et Prix Nobel de Médecine. Bull Soc Pathol Exot 92(5):287 [http://www.pathexo.fr/documents/articlesbull/T92-5-2078.pdf]

Tran D, Chastel C, Cenac A (1999) Paul-Louis Simond et la mission Marchoux au Brésil. Bull Soc Pathol Exot 92(5 Pt 2):388-91 [http:// www.pathexo.fr/documents/articles-bull/T92-5b-1963-PLS4.pdf] Chastel C (1999) La "peste" de Barcelone. Épidémie de fièvre jaune de 1821. Bull Soc Pathol Exot 92(5 Pt 2):405-7 [http://www.pathexo. fr/documents/articles-bull/T92-5b-1963-PLS3.pdf]

Guillet G, Leroy JP, Chastel C (1998) Lésions cutanées, risque viral et tiques d'oiseaux marins, un problème mondial; à propos d'une observation avec lésions pseudo-zoostériennes. Bull Soc Pathol Exot 91(2):160-3 [http://www.pathexo.fr/documents/articles-bull/T91-2MR96-015.pdf]

Chastel C (1998) André Dodin (1926-1995) : les années d'écoles militaires. Bull Soc Pathol Exot 91(5 Pt 1-2):361-2 [http://www. pathexo.fr/documents/articles-bull/T91-5-PF07.pdf]

Vermeil C, Marjolet M, Chastel C (1996) Argas et arbovirus : actualités. Bull Soc Pathol Exot. 89(5):363-5 [http://www.pathexo.fr/ documents/articles-bull/BullSocPatholExot-1996-89-5-363-365.pdf] Chastel C, Bailly-Choumara H, Bach-Hamba D, Le Lay G, Legrand MC, Le Goff F, Vermeil C (1995) Arbovirus transmis [http://www. pathexo.fr/documents/articles-bull/BullSocPatholExot-1995-88-3-081085.pdf]

Kles V, Michault A, Rodhain F, Mevel F, Chastel C. (1994) Enquêtes sérologiques concernant les arboviroses à Flaviviridae sur l'île de la Réunion (1971-1989). Bull Soc Pathol Exot 87(2):71-6 [http://www. pathexo.fr/documents/articles-bull/BullSocPatholExot-1994-87-2-7176.pdf]

Chastel C (1993) Actualité des fièvres hémorragiques zoonotiques d'Amérique du Sud. Bull Soc Pathol Exot 86(5 Pt 2):455-9 [http:// www.pathexo.fr/documents/articles-bull/BullSocPatholExot-1993-455459.pdf]
Leostic C, Masure O, Bietrix P, Le Flohic AM, Chastel C (1992) Le paludisme d'importation dans les hôpitaux de Brest de 1981 à 1990. Bull Soc Pathol Exot 85(5):365-7 [http://www.pathexo.fr/documents/ articles-bull/BullSocPatholExot-1992-365-367.pdf]

Le Lay-Rogues G, Arthur CP, Vanderwalle P, Hardy E, Chastel C (1990) Lapin de garenne, Oryctolagus cuniculus L. et arbovirus dans le sud-est de la France. Résultats de deux enquêtes sérologiques. Bull Soc Pathol Exot 83(4):446-56 discussion 456-7 [http://www.pathexo.fr/ documents/articles-bull/BullSocPatholExot-1990-446-457.pdf]

Humphery-Smith I, Grulet O, Le Lay G, Chastel C (1988) Pathogénicité de Spiroplasma sabaudiense (Mollicute) pour les cellules (C6/36) d'Aedes albopictus (Insecta: Diptera) in vitro. Bull Soc Pathol Exot Filiales 81(4):752-65 [http://www.pathexo.fr/documents/ articles-bull/BullSocPatholExot-1988-752-765.pdf]

Chabasse D, De Bray JM, Le Lay-Rogues G, Nedelec C, Chastel C (1986) Syndrome encéphalitique attribuable à un flavivirus observé au retour d'un voyage en Inde du Sud : dengue probable a expression neurologique. Bull Soc Pathol Exot Filiales 79(4):531-8 [http:// www.pathexo.fr/documents/articles-bull/BullSocPatholExot-1986531-538.pdf]

Doby JM, Chastel C, Couatarmanac'h A, Cousanca C, ChevrantBreton J, Martin A, Legay B, Guiguen C (1985) Questions étiologiques et épidémiologiques posées par l'érythème chronique migrant et la maladie de lyme. A propos de 4 observations réalisées au centre hospitalier régional de Rennes. Bull Soc Pathol Exot Filiales 78 (4):512-25 [http://www.pathexo.fr/documents/articles-bull/BullSocPatholExot-1985-512-525.pdf]

Chastel C, Guiguen C, Le Lay G, Monnat JY, Hardy E, Kerdraon G, Beaucournu JC (1985) Enquête sérologique arbovirus chez des oiseaux marins et non marins de Bretagne. Bull Soc Pathol Exot Filiales 78(5):594-605 [http://www.pathexo.fr/documents/articlesbull/BullSocPatholExot-1985-594-605.pdf]

Chastel C, Abalain-Colloc ML, Gilot B, Le Goff F, Gruffaz R, Simitzis AM (1985) Spiroplasmes et arthropodes hématophages; perspectives en pathologie tropicale. Bull Soc Pathol Exot Filiales. 78(5 Pt 2):769-79 [http://www.pathexo.fr/documents/articles-bull/BullSocPatholExot1985-769-779.pdf]

Masure O, Castel J, Reguer M, Bayon AM, Chastel C (1984) Le paludisme d'importation dans les hôpitaux de Brest de 1974 à 1980. Bull Soc Pathol Exot Filiales. 1984 May-Jun;77(3):298-304 [http:// www.pathexo.fr/documents/articles-bull/BullSocPatholExot-1984298-304.pdf]

Chastel C, Bach-Hamba D, Launay H, Le Lay G, Hellal H, Beaucournu JC (1983) Infections à arbovirus en Tunisie : nouvelle enquête sérologique chez les petits mammifères sauvages. Bull Soc Pathol Exot Filiales 76(1):21-33 [http://www.pathexo.fr/documents/articlesbull/BullSocPatholExot-1983-021-033.pdf]

Le Lay-Roguès G, Valle M, Chastel C, Beaucournu JC (1983) Petits mammifères sauvages et arbovirus en Italie. Bull Soc Pathol Exot Filiales 76(4):333-45 [http://www.pathexo.fr/documents/articles-bull/ BullSocPatholExot-1983-333-345.pdf]

Masure O, Bayon AM, Chastel C, Bergeret G (1983) Cryptococcose péritonéale : discussion d'un fait clinique. Bull Soc Pathol Exot Filiales 76(4):346-50 [http://www.pathexo.fr/documents/articles-bull/BullSocPatholExot-1983-346-350.pdf]

Chastel C, Launay H, Bailly-Choumara H, Le Lay G, Beaucournu JC (1982) Infections à arbovirus au Maroc : sondage sérologique chez les petits mammifères du nord du pays. Bull Soc Pathol Exot Filiales 75 (5):466-75 [http://www.pathexo.fr/documents/articles-bull/BullSocPatholExot-1982-466-475.pdf]

Chastel C, Bailly-Choumara H, Le Lay G (1981) Pouvoir pathogène naturel pour l'homme d'un variant antigénique du virus Soldado isolé au Maroc. Bull Soc Pathol Exot Filiales 74(5):499-505 [http:// www.pathexo.fr/documents/articles-bull/BullSocPatholExot-1981499-505.pdf] 
Chastel C, Launay H, Rogues G, Beaucournu JC (1980) Infections à arbovirus en Espagne: enquête sérologique chez les petits mammifères. Bull Soc Pathol Exot Filiales. Jul-Aug;73(4):384-90 [http:// www.pathexo.fr/documents/articles-bull/BullSocPatholExot-1980384-390.pdf]

Chastel C, Roguès G (1980) Ecologie du virus Soldado: absence apparente d'un inhibiteur du virus dans les tissus de la tique vectrice Ornithodoros (Alectorobius) maritimus Vermeil et Marguet 1967. Bull Soc Pathol Exot Filiales 73(4):399-405 [http://www.pathexo.fr/ documents/articles-bull/BullSocPatholExot-1980-399-405.pdf]

Chastel C, Rogues G, Beaucournu-Saguez F, Hellal H, Le Goff F, Beaucournu JC (1977) Enquête séro-épidémiologique mixte arbovirus-arénavirus chez les petits mammifères de Tunisie. Bull Soc Pathol Exot Filiales 70(5):471-9 [http://www.pathexo.fr/ documents/articles-bull/BullSocPatholExot-1977-471-479.pdf]

Thomas J, Josserand C, Chastel C, Sagnet H, Lassale Y, Le Vourch C (1973) Atteinte cardiovasculaire lors du kwashiorkor. Etude clinique, radiologique, électrocardiographique et anatomique (à propos de 655 cas). Bull Soc Pathol Exot Filiales 66(3):426-34

Thomas J, Chastel C, Forcain L (1970) Latence clinique et parasitaire dans les filarioses à Loa loa et à Onchocerca volvulus. Bull Soc Pathol Exot Filiales 63(1):90-4 [http://www.pathexo.fr/documents/ articles-bull/BullSocPatholExot-1970-390-394.pdf]

Chastel C, Nicoli RM, Quilici M, Revil H, Mafart Y (1969) Une nouvelle observation de sporozoaire Incertae sedis. Bull Soc Pathol Exot Filiales 62(1):126-31 [http://www.pathexo.fr/documents/articles-bull/ BullSocPatholExot-1969-126-131.pdf]

Chastel C, Brottes H, Goasguen J, Le Mao G, Favre C, Thomas J, Sagnet H (1969) Etats de malnutrition protidique et de dénutrition des enfants biafrais. Etude anatomo-pathologique. Bull Soc Pathol Exot Filiales 62(5):941-55 [http://www.pathexo.fr/documents/ articles-bull/BullSocPatholExot-1969-941-955.pdf]

Chastel C, Thomas J (1968) Elliptocytose constitutionnelle et paludisme. Bull Soc Pathol Exot Filiales 61(4):605-13 [http://www. pathexo.fr/documents/articles-bull/BullSocPatholExot-1968-605613.pdf]
Chastel C, Rageau J, Abonnenc E (1966) Présence de Culicoides anophelis Edwards, 1922 (Diptera : Ceratopogonidae) au Cambodge. Bull Soc Pathol Exot Filiales 59(1):151-5 [http://www.pathexo.fr/ documents/articles-bull/BullSocPatholExot-1966-151-155.pdf]

Chastel C (1965) Infections humaines au Cambodge par le virus TH 36 (« Dengue type $5 »)$ ou un agent étroitement apparenté. Bull Soc Pathol Exot Filiales 58(6):1007-17 [http://www.pathexo.fr/ documents/articles-bull/BullSocPatholExot-1965-1007-1017.pdf]

Chastel C (1964) Infections humaines au Cambodge par le virus Chikungunya ou un agent étroitement apparenté. III. - Épidémiologie. Bull Soc Pathol Exot Filiales 57(1):65-82 [http://www.pathexo.fr/ documents/articles-bull/BullSocPatholExot-1964-065-082.pdf]

Chastel C (1963) Infections humaines au Cambodge par le virus Chikungunya ou un agent étroitement apparenté. I. - Clinique. Isolements et identification des virus. Sérologie. Bull Soc Pathol Exot Filiales 56(5):892-915 [http://www.pathexo.fr/documents/articlesbull/BullSocPatholExot-1963-892-915.pdf]

Chastel C (1963) Infections humaines au Cambodge par le virus Chikungunya ou un agent étroitement apparenté. II. - Anatomie pathologique expérimentale. Bull Soc Pathol Exot Filiales 56(5):915-24 [http://www.pathexo.fr/documents/articles-bull/BullSocPatholExot1963-915-924.pdf]

Chastel C, Ridet J (1960) Rickettsioses et néorickettsioses en HauteVolta. Intérêt de la microagglutination des rickettsies sur lame en zone intertropicale. Bull Soc Pathol Exot Filiales 53(1):180-210 [http://www.pathexo.fr/documents/articles-bull/BullSocPatholExot1960-180-210.pdf]

Chastel C (1960) Deux cas d'elliptocytose constitutionnelle observés chez des africains en Haute-Volta. Bull Soc Pathol Exot Filiales 53(2):357-65 http://www.pathexo.fr/documents/articles-bull/BullSoc PatholExot-1960-357-365.pdf]

Coudert J, Chastel C (1952) Note sur la morphologie du Plasmodium berghei Vincke et Lips observé au microscope en contraste de phase. Bull Soc Pathol Exot Filiales 45(6):740-3 [http://www.pathexo.fr/ documents/articles-bull/BullSocPatholExot-1952-740-743.pdf] 